ARTICLE

https://doi.org/10.1038/s41467-019-12488-9

\title{
Evaporative electron cooling in asymmetric double barrier semiconductor heterostructures
}

\author{
Aymen Yangui (1) 1,2,4, Marc Bescond ${ }^{1,2,4}$, Tifei Yan ${ }^{1}$, Naomi Nagai ${ }^{1} \&$ Kazuhiko Hirakawa $a^{1,2,3 \star}$
}

Rapid progress in high-speed, densely packed electronic/photonic devices has brought unprecedented benefits to our society. However, this technology trend has in reverse led to a tremendous increase in heat dissipation, which degrades device performance and lifetimes. The scientific and technological challenge henceforth lies in efficient cooling of such highperformance devices. Here, we report on evaporative electron cooling in asymmetric Aluminum Gallium Arsenide/Gallium Arsenide (AIGaAs/GaAs) double barrier heterostructures. Electron temperature, $T_{\mathrm{e}}$, in the quantum well (QW) and that in the electrodes are determined from photoluminescence measurements. At $300 \mathrm{~K}, T_{\mathrm{e}}$ in the $\mathrm{QW}$ is gradually decreased down to $250 \mathrm{~K}$ as the bias voltage is increased up to the maximum resonant tunneling condition, whereas $T_{\mathrm{e}}$ in the electrode remains unchanged. This behavior is explained in term of the evaporative cooling process and is quantitatively described by the quantum transport theory.

\footnotetext{
${ }^{1}$ Institute of Industrial Science, University of Tokyo, 4-6-1 Komaba, Meguro-ku, Tokyo 153-8505, Japan. ${ }^{2}$ LIMMS/CNRS-IIS, UMI 2820, 4-6-1 Komaba, Meguro-ku, Tokyo 153-8505, Japan. ${ }^{3}$ Institute for Nano Quantum Information Electronics, University of Tokyo, 4-6-1 Komaba, Meguro-ku, Tokyo 153-8505, Japan. ${ }^{4}$ These authors contributed equally: Aymen Yangui, Marc Bescond. *email: hirakawa@iis.u-tokyo.ac.jp
} 
$\mathrm{N}$ anoscale miniaturization of semiconductor devices has enabled ultrahigh-density integration and ultrafast operation of transistors and optoelectronic devices. A significant and sustained upward trend of nanoscale miniaturization is expected for the coming years owing to tremendous growth of the so-called "new communication and information technologies". However, this downscaling of devices has brought technological issues. High electric fields in such nanoscale devices generate hot carriers, which transfer their kinetic energies to the lattice, leading to the formation of hot spots ${ }^{1-3}$. The on-chip power density now exceeds $100 \mathrm{~W} \mathrm{~cm}^{-2} 2,4$ leading to lattice temperature above $400 \mathrm{~K}^{5}$. These self-heating effects result in significant reduction in performances ${ }^{6}$ and lifetimes of the devices. Moreover, the refrigeration of the entire systems is extremely power consuming $3,7,8$. Accordingly, efficient integrated cooling solutions are listed in the International Roadmap for Devices and Systems as the top five long-term key challenges to address 9 . The engineering of efficient cooling is then one of the major scientific, technological, and environmental tasks in a context of energy resource shortage ${ }^{10,11}$.

The most commonly used solid-state refrigeration is based on the thermoelectric Peltier effect ${ }^{12}$. In the thermoelectric regime, electrons frequently experience scattering, leading to the degradation in the thermoelectric power factor $S^{2} \sigma$, where $S$ and $\sigma$ are the Seebeck coefficient and the electrical conductivity, respectively. Furthermore, the materials used to obtain efficient Peltier effect such as BiTe are not compatible with the standard semiconductor fabrication processes.

Another interesting mechanism for solid-state refrigeration is the thermionic cooling ${ }^{13}$. Electrons thermionically emitted from the cathode transfer their kinetic energies to the anode and give rise to refrigeration in the cathode ${ }^{14}$. In 1990's, the idea of thermionic cooling revived and semiconductor heterostructure refrigerators based on this concept were investigated ${ }^{15-20}$. Lattice cooling by as much as 1 to $3 \mathrm{~K}$ was observed at $300 \mathrm{~K}$ by using a single or multiple barrier structures ${ }^{21-25}$.

An alternative approach to reduce the hotspot effect is to directly cool down electrons before electrons transfer their kinetic energy to the lattice. The aim of the present work is to demonstrate that a significant electron cooling as much as $50 \mathrm{~K}$ is possible in a semiconductor heterostructure operating at room temperature. The studied Aluminum Gallium Arsenide/Gallium Arsenide (AlGaAs/GaAs) asymmetric double-barrier heterostructure, which combines resonant tunneling and thermionic emission, was originally proposed by Chao et al. as a lattice cooler $^{26}$. Here, we demonstrate that this heterostructure is also very efficient for cooling the electron system in the quantum well (QW). The electron temperature, $T_{\mathrm{e}}$, in the QW as well as $T_{\mathrm{e}}$ in the electrodes are determined from photoluminescence (PL) measurements. At $300 \mathrm{~K}, T_{\mathrm{e}}$ in the $\mathrm{QW}$ is remarkably reduced by as much as $50 \mathrm{~K}$ as the bias voltage is increased up to the maximum resonant tunneling condition. This behavior is qualitatively explained in terms of the evaporative cooling process, which is well-known in the field of the cold atom physics ${ }^{27}$. In this work, we have implemented the concept of the evaporative cooling in a solid-state system, i.e., the semiconductor heterostructures, and observed a significant electron cooling as much as $50 \mathrm{~K}$ at $300 \mathrm{~K}$. The observed cooling behavior is quantitatively confirmed by quantum transport calculations that self-consistently couples the non-equilibrium Green's function (NEGF) formalism for electrons with the heat equation (see Supplementary Methods).

\section{Results}

Asymmetric double-barrier heterostructures. The samples used in the present work were grown by molecular beam epitaxy and prepared by growing successively on an n-type GaAs substrate, a 300-nm-thick n-GaAs emitter layer (Si: $1 \times 10^{17} \mathrm{~cm}^{-3}$ ), a 5-nmthick undoped GaAs layer, an undoped 15-nm-thick $\mathrm{Al}_{0.4} \mathrm{Ga}_{0.6} \mathrm{As}$ barrier (we call this barrier "the emitter barrier" hereafter), an undoped 4-nm-thick GaAs QW, an undoped 100-nm-thick $\mathrm{Al}_{0.25} \mathrm{GaAs}_{0.75} \mathrm{As}$ barrier (we call this barrier "the collector barrier" hereafter), and a 200-nm-thick n-GaAs collector layer (Si: $\left.1 \times 10^{17} \mathrm{~cm}^{-3}\right)$. We used a rather thick emitter barrier $(15 \mathrm{~nm})$ to quantum-mechanically decouple the electronic states in the QW from those in the emitter electrode and separate the photoluminescence (PL) in the QW from that in the electrodes. Thinner barrier would also degrade the heat insulation between the electrons in the QW and those of the emitter, leading to a reduction of the electron cooling. The wafer was then patterned into $200 \times 200 \mu \mathrm{m}^{2}$ mesas by photolithography. 70/50-nm-thick $\mathrm{AuGeNi} / \mathrm{Au}$ contacts were deposited on the front and back sides of the mesa. For optical measurements, we made a $50 \times 50 \mu \mathrm{m}^{2}$ window on the top of each mesa, where we deposited semitransparent 5-nm-thick $\mathrm{NiCr}$ film to ensure the uniformity of the applied bias voltage. The wafer was finally annealed at $450{ }^{\circ} \mathrm{C}$ in Ar ambient for $30 \mathrm{~s}$. For more detail, see the Supplementary Note 1 and the Supplementary Fig. 1.

Figure 1a illustrates the band diagram of the asymmetric double-barrier heterostructure. A positive voltage is applied to the collector electrode with respect to the emitter electrode. Electrons are injected from the emitter electrode into the quantized

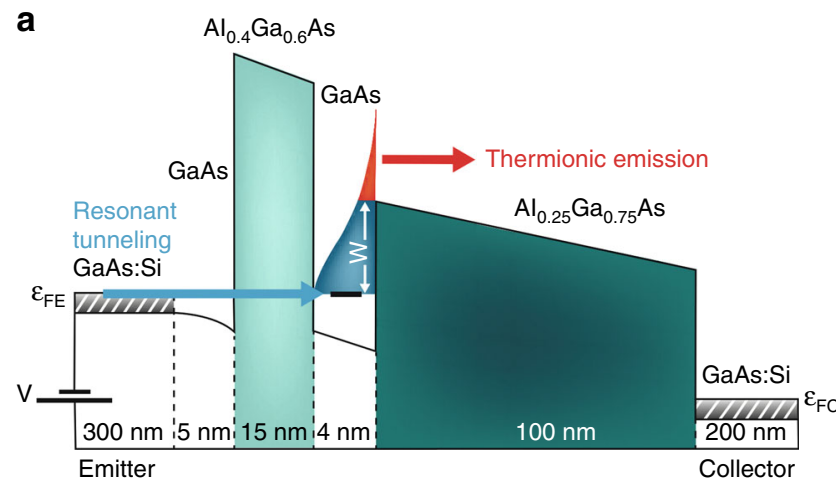

b

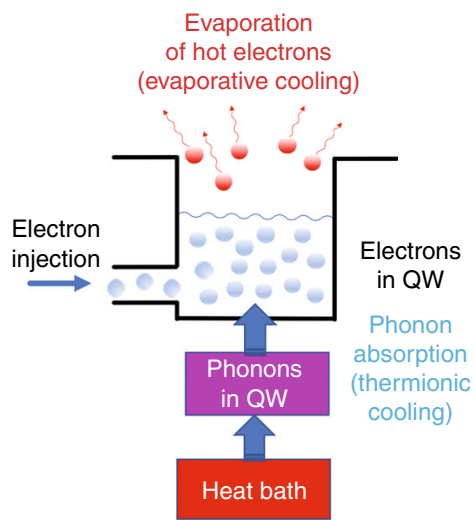

Fig. 1 The asymmetric double-barrier heterostructure for evaporative electron cooling. a Band diagram of the asymmetric double-barrier heterostructure. Electron transport mechanism, i. e., the resonant tunneling through the thinner barrier (blue arrow) and the thermionic emission above the thicker barrier (red arrow), is schematically shown. b Schematic diagram for the heat flow in the electron-lattice system in the heterostructure. The concepts of the evaporative and thermionic cooling are schematically illustrated 

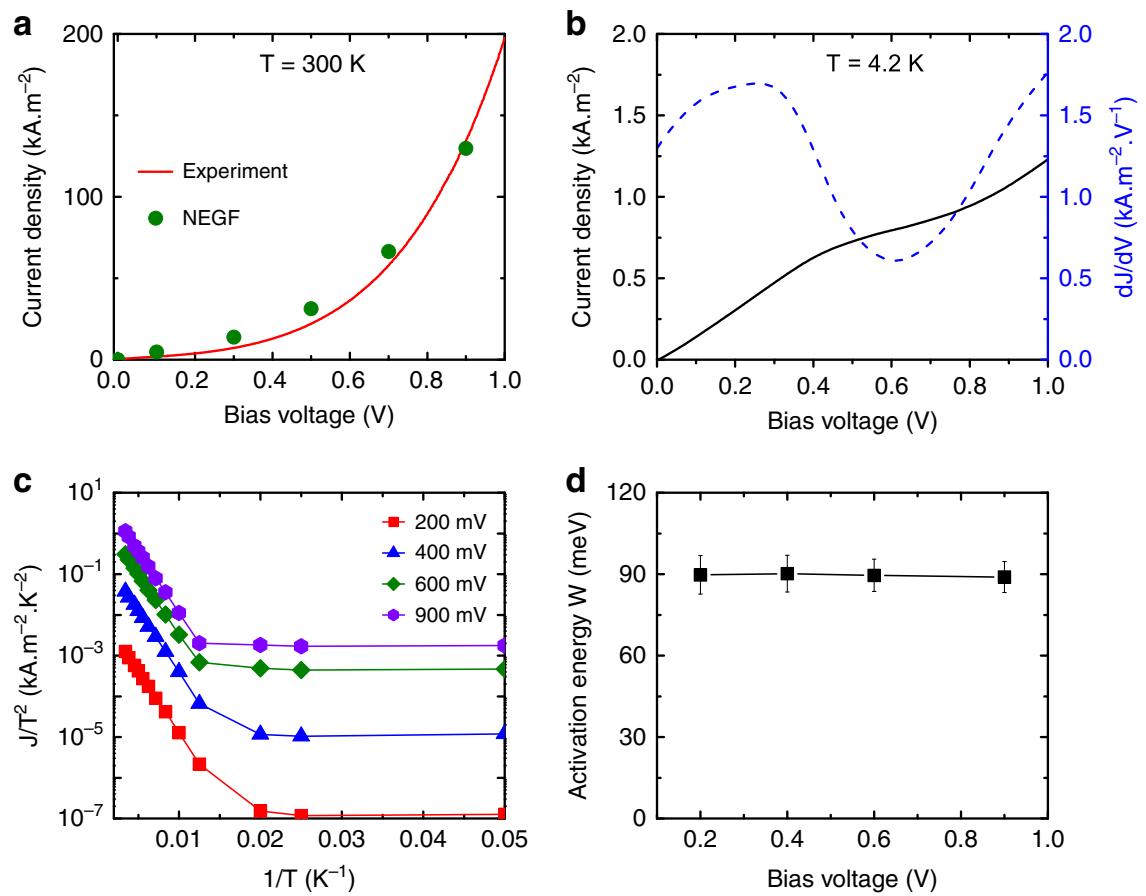

Fig. 2 Electron transport measurements. a Current density $J$ measured as a function of the bias voltage $V$ at $300 \mathrm{~K}$ (red solid line). The green symbols are the calculated current densities by NEGF simulations. $\mathbf{b} \mathrm{J}-\mathrm{V}$ curve measured at $4.2 \mathrm{~K}$ (black solid line). The resonant tunneling shoulder can be clearly identified at around $V \sim 0.5 \mathrm{~V}$. The dotted curve shows the differential conductance curve, $\mathrm{dl} / \mathrm{d} V . \mathbf{c} \log \left(J / T^{2}\right)$ is plotted as a function of $1 / T$ at various $V$. d Thermal activation energy, $W$, determined from the $J-T$ data shown in (c)

subband in the QW by resonant tunneling through the emitter barrier. Due to the very thick collector barrier, electrons injected into the QW cannot tunnel out to the collector electrode; instead, electrons whose kinetic energy is larger than the height of the collector barrier are removed by thermionic emission. This effect leads to the cutoff of the high-energy tail of the distribution function of electrons in the QW which then thermalized into a new quasi-equilibrium state at a lower temperature. This is the so-called evaporative cooling 28,29 .

Figure 1b schematically illustrates how the non-equilibrium electron and lattice systems in the QW interact with each other. The electron system is cooled through "evaporation" over the collector barrier. The colder electron system in the QW is in close contact with the warmer lattice system via electron-phonon interaction. As a result, the electron system is warmed up by the lattice system through phonon absorption, while the lattice system in the QW is cooled down by the electron system ("thermionic cooling"). Furthermore, since the QW is contacted by the electrode layers, there is a heat flow from the heat bath to the QW.

Determination of the electron temperature. To characterize the sample structure, we first measured its current-voltage characteristics. Figure 2 a plots the current density, $J$, measured as a function of the bias voltage, $V$, at $300 \mathrm{~K}$. As seen in this figure, the $J-V$ curve at $300 \mathrm{~K}$ is rather featureless and $J$ gradually increases with increasing $V$. This $J-V$ curve is perfectly reproduced by the NEGF quantum transport calculation. To gain more insight to the resonant tunneling electron injection, we also measured $J$ at $4.2 \mathrm{~K}$ (see Fig. 2b). A kink due to the shut-off of the resonant tunneling process can be identified near $0.5 \mathrm{~V}$, which is more clearly visible in the plot of $\mathrm{d} I / \mathrm{d} V$. This confirms the resonant tunneling injection of electrons into the QW. Figure $2 \mathrm{c}$ plots the temperature dependence of $J$ measured at various $V$. An exponential decrease in $J$ with decreasing $T$ is observed for $T>100 \mathrm{~K}$, whereas $J$ becomes almost temperature-independent for $T<50 \mathrm{~K}$, indicating that the current is carried by tunneling. The hightemperature behavior is dominated by the thermionic emission process $^{30}$, whose magnitude is proportional to $T^{2} \exp \left(-W / k_{\mathrm{B}} T\right)$. Here, $W$ is the activation energy (see Fig. 1a) and $k_{\mathrm{B}}$ the Boltzmann constant. From the slope of the $\log \left(J / T^{2}\right)$ vs $1 / T$ plots at high temperatures, we estimated $W$ (Fig. 2 d). $W$ does not depend on $V$ and stays at around $\sim 90 \mathrm{meV}$, which is consistent with the designed band structure.

Let us discuss the electron temperature, $T_{\mathrm{e}}$, in the QW. For this purpose, we carried out PL measurements at $300 \mathrm{~K}$. The measurements were performed by using an excitation photon energy of $2.54 \mathrm{eV}$. This rather high photon energy is to reduce the penetration depth of the excitation light into GaAs (it is $\sim 100 \mathrm{~nm}$ at $2.54 \mathrm{eV}$ ) and have a higher PL sensitivity in the shallow QW region. The excitation power was set to be $2 \mathrm{~mW}$ and the spot size was about $10 \mu \mathrm{m}$. Figure $3 \mathrm{a}$ plots the PL spectra measured at various $V$ at $300 \mathrm{~K}$. A PL peak is observed at $\sim 1.43 \mathrm{eV}$, which results from the emission in the $\mathrm{n}-\mathrm{GaAs}$ electrodes. A shaper $\mathrm{PL}$ peak is observed at $1.552 \mathrm{eV}$, together with a shoulder at $1.578 \mathrm{eV}$. The peak at $1.552 \mathrm{eV}$ originates from the radiative recombination of the ground subband electrons in the QW with heavy holes $(\mathrm{HH})$ and the shoulder at $1.578 \mathrm{eV}$ is due to the electron-light hole (LH) emission.

Assuming the Maxwell-Boltzmann distribution for electrons, $T_{\mathrm{e}}$ in the QW as well as that in the electrodes were deduced from the high-energy tail of the PL spectra ${ }^{31-33}$. In the electrode regions, we have a three-dimensional density of states and the PL intensity can be approximately expressed as,

$$
I_{\mathrm{el}} \propto \sqrt{h \nu-E_{\mathrm{g}}} \exp \left(-\frac{h \nu-E_{\mathrm{g}}}{k_{\mathrm{B}} T_{\mathrm{e}}}\right),
$$

where $I_{\mathrm{el}}$ is the PL intensity of the electrode emission, $h v$ the 

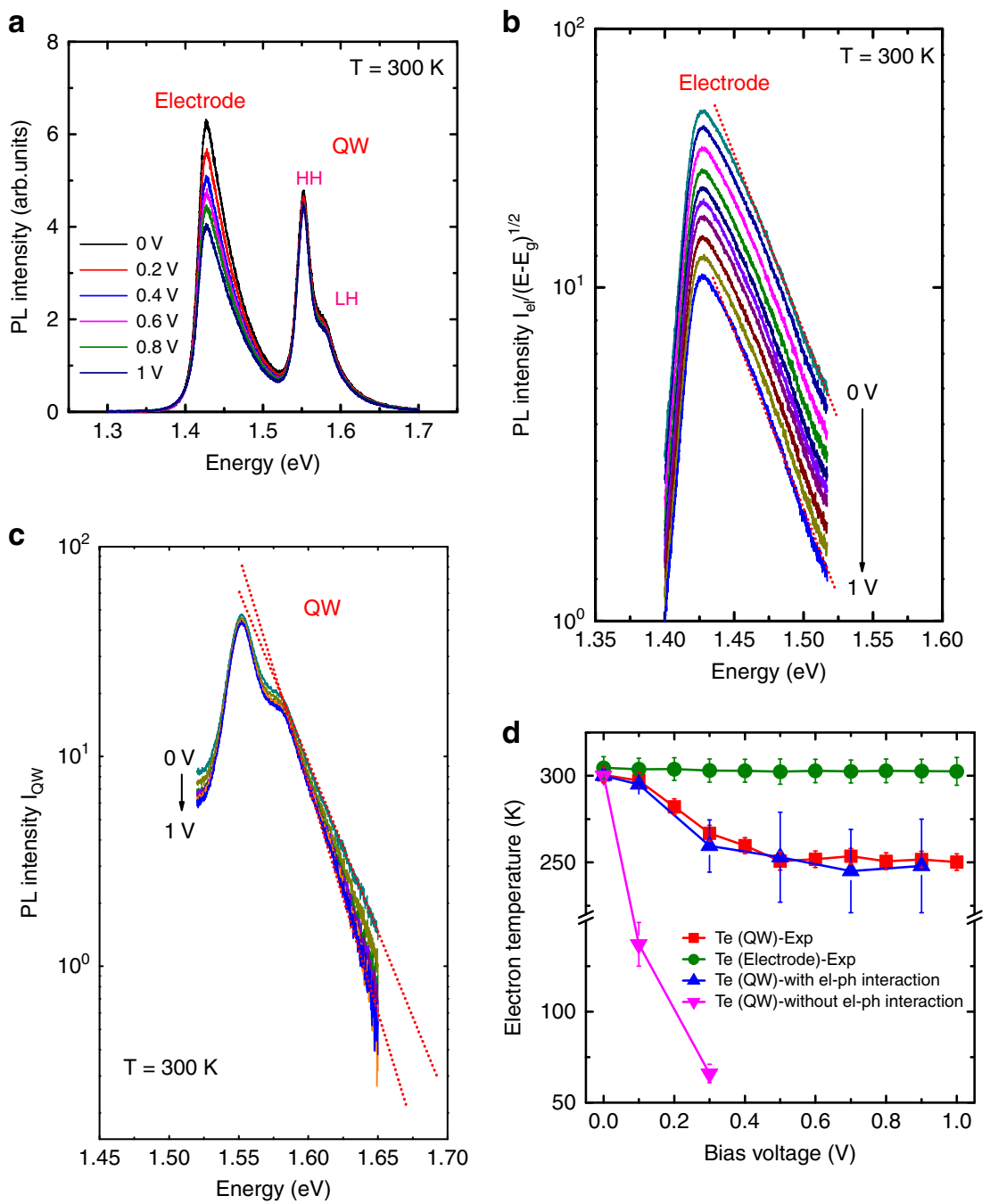

Fig. 3 Electron temperature determined from photoluminescence measurements. a Photoluminescence (PL) spectra measured at $300 \mathrm{~K}$ for various bias voltages. Peaks corresponding to the recombination of electrons in the quantum well (QW) with heavy holes (HH) and light holes (LH) are also highlighted. $\mathbf{b}$ The plot of the PL intensity of the electrode, in a logarithmic scale, as a function of the photon energy shows that the slope of the high-energy tail is bias independent. The red dotted lines are eyeguides. c The intensity of the QW PL emission as a function of the photon energy shows that the highenergy tail of the PL spectra becomes steeper as the bias voltage is increased. $\mathbf{d}$ The electron temperatures in the QW (red squares) and electrode (green circles) determined from PL measurements are plotted as a function of the bias voltage. The blue triangles denote the electron temperature calculated by taking into account electron-phonon interaction, showing a good agreement with the experimental data (red squares). The pink triangles are the electron temperatures calculated by suppressing the electron-phonon interaction

photon energy, $E_{\mathrm{g}}$ the bandgap of GaAs, and $k_{\mathrm{B}}$ is the Boltzmann constant. As for the PL from the QW, we have constant twodimensional densities of states for electrons and holes. Then, we have

$$
I_{\mathrm{QW}} \propto \exp \left(-\frac{h \nu-E_{0}}{k_{\mathrm{B}} T_{\mathrm{e}}}\right),
$$

where $I_{\mathrm{QW}}$ is the $\mathrm{PL}$ intensity of the $\mathrm{QW}$ region, and $E_{0}$ the energy difference between the electron-hole quantized ground subband. Using Eqs. (1) and (2), we replotted in Fig. 3b, c the PL intensities for the electrode and the QW, respectively. Figure $3 \mathrm{~b}$ plots $\log \left[I_{\mathrm{el}} / \sqrt{h \nu-E_{\mathrm{g}}}\right]$ as a function of $h v$ for the electrode emission. The high-energy exponential thermal tail of the PL is clearly observed and $T_{\mathrm{e}}$ in the electrode is deduced from its slope. The determined $T_{\mathrm{e}}$ is $\sim 305 \mathrm{~K}$ and remains almost unchanged when $V$ is varied, as shown in Fig. $3 \mathrm{~d}$ (green circles). Concerning the PL from the QW, we plot $\log I_{\mathrm{QW}}$ as a function of $h v$ in Fig. $3 \mathrm{c}$ and determine $T_{\mathrm{e}}$ from the slope of its high-energy tail. As seen in the figure, the slope becomes steeper as $V$ is increased, reflecting the reduction in $T_{\mathrm{e}}$ in the QW. In Fig. 3d, the obtained $T_{\mathrm{e}}$ for the QW are plotted by red squares as a function of $V$. Note that $T_{\mathrm{e}}$ in the QW is reduced from room temperature by as much as $50 \mathrm{~K}$ when $V=0.5 \mathrm{~V}$. For $V$ above $0.5 \mathrm{~V}, T_{\mathrm{e}}$ in the $\mathrm{QW}$ almost saturates.

As described before, the decrease in $T_{\mathrm{e}}$ in the QW results from evaporation of electrons ${ }^{28,29,34}$ and can be explained as follows; at $V=0$, electrons in the QW are in the thermal equilibrium with those in the emitter and collector electrodes. The local net current vanishes and the electrons in the QW have the same thermal distribution as those in the electrodes, which are at room temperature. Applying a positive bias induces a net flow of electrons from the emitter to the collector. In the $\mathrm{QW}$, the thermally excited carriers that have an energy higher than the collector barrier are driven outside the QW toward the collector. The electrons remaining in the QW are re-thermalized 
within a few tens of femtoseconds to a new quasi-equilibrium distribution at a lower temperature via electron-electron and electron-phonon scattering. The increase in $V$ promotes the current flow, i.e., electron evaporation, until the maximal resonant tunneling is reached at $V=0.5 \mathrm{~V}$. For $V$ above $0.5 \mathrm{~V}$, the subband in the QW goes below the conduction band edge in the emitter and the resonant tunneling injection should be shut off. However, as shown in Fig. 2b, the measured current does not decrease but it becomes almost constant. This is probably due to scattering-assisted injection of electrons from the emitter to the QW. Figure 3d shows an excellent agreement between experiment and quantum transport calculations. We note that electrons in the collector are heated up by the landing of hot electrons that escape from the QW. However, since the heat released by the hot electrons are very quickly redistributed and shared among very many electrons in the collector electrode, the increase in the electron temperature in the collector is expected to be much smaller (below $0.1 \mathrm{~K}$ ). Furthermore, the surface metal electrode also helps good thermal anchoring of electrons in the collector electrode.

At this point, we would like to discuss interactions between the electron and the lattice systems. In polar materials such as GaAs, interactions between electrons and lattice are mainly mediated by polar optical (PO) phonons ${ }^{35-37}$. Electrons injected onto the QW state are then subject to $\mathrm{PO}$ phonon absorption. This transfer of energy from the lattice to the electron system warms up the electron system. To see this effect, we plot, in Fig. $3 \mathrm{~d}, T_{\mathrm{e}}$ in the QW obtained from quantum transport calculations in which the interaction between the electrons and the PO phonons in the QW is intentionally suppressed. It shows that the calculated reduction in $T_{\mathrm{e}}$ is significantly enhanced; for instance, $T_{\mathrm{e}}$ is $137 \mathrm{~K}$ at $V=0.1 \mathrm{~V}$. For higher voltages (i.e. $V$ superior than $0.5 \mathrm{~V}$ ), the calculation of $T_{\mathrm{e}}$ cannot be numerically performed, because the present theoretical model assumes the acoustic phonon scattering to be elastic and, because of this reason, the quasi-localized state becomes inaccessible once it goes below the conduction band edge of the emitter.

Efficiency of lattice cooling. Finally, let us make a comment on the lattice refrigeration effect. As previously described, phonon absorption by colder electrons in the QW cools lattice (thermionic cooling). Since the determination of the local lattice temperature in the QW is very challenging and out of the scope of the present paper, we want to make a theoretical estimation. Figure $4 \mathrm{a}$ shows the calculated cooling power density per unit area, $J_{\mathrm{Q}}$, as a function of $V . J_{\mathrm{Q}}$ is determined in NEGF from the

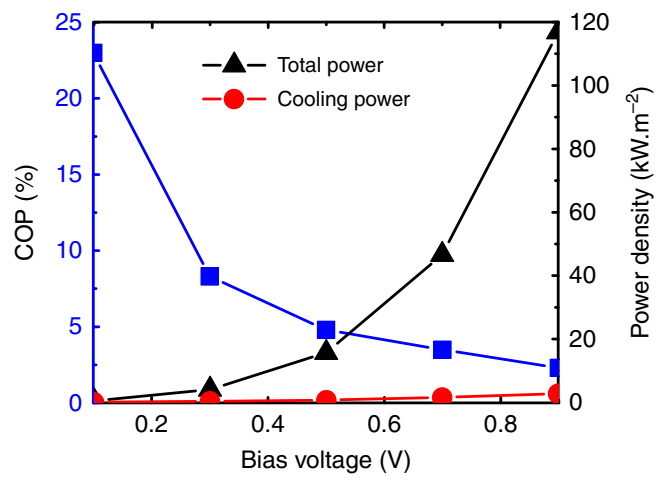

Fig. 4 Cooling power density. The cooling power density (red circles) and the input electrical power density applied to the heterostructures (black triangles) as a function of the bias voltage. The coefficient of performance (COP) is then obtained from the ratio of these two quantities (blue squares) spatial derivative of the electronic energy flow and defines the power transferred from the lattice to the electrons (see Supplementary Methods for more details) ${ }^{38}$. We see that $J_{\mathrm{Q}}$ increases with $V$. It goes from $111 \mathrm{~W} \mathrm{~m}^{-2}$ at $0.1 \mathrm{~V}$ to $2.7 \mathrm{~kW} \mathrm{~m}^{-2}$ at $0.9 \mathrm{~V}$. However, the total input electrical power density, $J V$, applied to the heterostructure also strongly increases. A meaningful criterion is then given by the coefficient of performance, COP $\equiv J_{\mathrm{Q}} / J V$, which is also plotted in Fig. 4a. Interestingly, the COP shows a maximum at a low bias voltage (COP $\sim 23 \%$ for $V=0.1 \mathrm{~V})$ and continuously decreases with increasing $V$. This feature results from the enhancement of the escape rate of electrons from the QW at high electric fields, hence reducing the interaction with phonons. Moreover, in this regime, the strong increase in the current component which directly goes above the collector barrier also contributes to the COP reduction. Figure 4 emphasizes the trade-off between a high COP, which is usually obtained near the equilibrium state, and a large cooling power, which is obtained at larger $V$.

\section{Discussion}

Despite a good COP value, theory estimates a rather weak lattice temperature cooling, in the order of a few $\mathrm{mK}$. The reason for such a small lattice cooling effect is threefold: the first is the difference in the specific heats of electrons and phonons. Indeed the specific heat of phonons in GaAs is, at room temperature, equal to $46 \mathrm{Jmol}^{-1} \mathrm{~K}^{-1}$, while the one for electrons is several orders of magnitude smaller ${ }^{39}$. The second is the high thermal conductance of the AlGaAs emitter and collector barriers. Since the thin QW is sandwiched between the emitter and the collector electrode layers, heat flow from the electrodes is significant. Third, we adopted a relatively low doping density in the electrode to reduce the peak width of the electrode PL (as shown in Fig. 3a).

For more significant lattice cooling, we need to increase the current density and realize a cooling power density of the order of $10^{3} \mathrm{~W} \mathrm{~cm}^{-3}$ in the QW. This value can be achieved by increasing the doping density in the emitter electrode and by reducing the thickness of the emitter barrier. Theoretical investigations reported in ref. ${ }^{40}$ have shown that a structure with a higher doping level $\left(10^{18} \mathrm{~cm}^{-3}\right)$ in the emitter and a thinner emitter barrier $(2.4 \mathrm{~nm})$ can achieve a maximum COP above 100\% (In the present cooling device structure, heat is removed from the QW and transferred to the collector region. Therefore, the COP estimated only for the QW region can exceed 100\%) at $V=0.1 \mathrm{~V}$, with a cooling power density of $2 \times 10^{2} \mathrm{~W} \mathrm{~cm}^{-2}$. We are convinced that fully optimized structures will be able to locally refrigerate the lattice by a few tens degree celsius under typical operating conditions.

In this paper, we have investigated both experimentally and theoretically the cooling properties of $\mathrm{AlGaAs} / \mathrm{GaAs}$ asymmetric double-barrier heterostructures. We have shown that electrons in the QW are refrigerated by the "evaporative cooling" process. Electron temperatures in the QW and in the electrodes were determined by PL measurements. We have found that, when operated at room temperature, $T_{\mathrm{e}}$ in the QW decreases down to $250 \mathrm{~K}$ with increasing the bias voltage up to the maximum resonant tunneling condition. The experimental results have been well explained by quantum transport theory. Furthermore, we have discussed the interplay between the electron and lattice cooling in the QW. The lattice system is refrigerated by the "thermionic cooling" process and its COP is in the order of 5-20\%. These results make our heterostructure device promising for a comprehensive heat management in nanodevices.

\section{Methods}

Sample fabrication. A detailed description of the sample fabrication is provided in the Supplementary Notes 1 and 2 and the Supplementary Fig. 1. 
Electron temperature determination. The determination of electron temperatures from the fit of the high-energy tail of the PL spectra, assuming the Maxwell-Boltzmann distribution, has been well established and has often been used particularly for GaAs/AlGaAs heterostructures ${ }^{31-33}$. However, it is worth mentioning that the calculation of the electron temperature directly from the slope of high-energy tail of PL spectra relies on several approximations. Full details of the approximations used are provided in the Supplementary Note 3.

Quantum transport code. Quantum transport code, including the calculations of current characteristics, electron temperatures, and coefficients of performance, can be found in the Supplementary Methods and in ref. ${ }^{40}$.

\section{Data availability}

The data that support the plots within this paper and other findings of this study are available from the corresponding author upon reasonable request.

The source data underlying Figs. $2 \mathrm{a}-\mathrm{d}, 3 \mathrm{a}-\mathrm{d}$, and 4 are provided as a Source Data file.

\section{Code availability}

The quantum transport code used for the calculations of current characteristics, electron temperatures and coefficients of performance are available from the corresponding author upon reasonable request.

Received: 7 January 2019; Accepted: 3 September 2019; Published online: 03 October 2019

\section{References}

1. Tenbroek, B. M., Lee, M. S. L., Redman-White, W., Bunyan, J. T. \& Uren, M. J. Self-heating effects in SOI MOSFETs and their measurement by small signal conductance techniques. IEEE Trans. Electron Devices 43, 2240-2248 (1996).

2. Gaska, R., Osinsky, A., Yang, J. W. \& Shur, M. S. Self-heating in high-power AlGaN-GaN HFETs. IEEE Electron Device Lett. 19, 89-91 (1998).

3. Pop, E., Sinha, S. \& Goodson, K. E. Heat generation and transport in nanometer-scale transistors. Proc. IEEE 94, 1587-1601 (2006).

4. Pop, E. \& Goodson, K. E. Thermal phenomena in nanoscale transistors. J. Electron. Packaging 128, 102-108 (2006).

5. Rhyner, R. \& Luisier, M. Minimizing self-heating and heat dissipation in ultrascaled nanowire transistors. Nano Lett. 16, 1022-1026 (2016).

6. Srinivasan J., Adve S. V., Bose P., Rivers J. A. International Conference on Dependable Systems and Networks (IEEE, 2004).

7. Pedram, M. \& Nazarian, S. Thermal modeling, analysis, and management in VLSI circuits: principles and methods. Proc. IEEE 94, 1487-1501 (2006).

8. Zhang, H., Shao, S., Xu, H., Zou, H. \& Tian, C. Free cooling of data centers: a review. Renew. Sust. Energy Rev. 35, 171-182 (2014).

9. International Roadmap for Devices and Systems. https://irds.ieee.org/ roadmap-2017 (2017).

10. Meijer, G. I. Cooling energy-hungry data centers. Science 328, 318-319 (2010).

11. Wang, X., Vasilakos, A. V., Chen, M., Liu, Y. \& Kwon, T. T. A survey of green mobile networks: opportunities and challenges. Mob. Netw. Appl. 17, 4-20 (2012).

12. Kretzschmar, K. M. \& Wilkie, D. R. The use of the peltier effect for simple and accurate calibration of thermoelectric devices. Proc. R. Soc. Lond. B Biol. Sci. 190, 315-321 (1975)

13. Murphy, E. L. \& Good, R. H. Thermionic emission, field emission, and the transition region. Phys. Rev. 102, 1464-1473 (1956).

14. Hatsopoulos G., Gyftopoulos E. Thermionic Energy Conversion. Vol. II. Theory, technology, and application (MIT Press, Cambridge, MA, 1979).

15. Mahan, G. D. Thermionic refrigeration. J. Appl. Phys. 76, 4362-4366 (1994).

16. Shakouri, A. \& Bowers, J. E. Heterostructure integrated thermionic coolers. Appl. Phys. Lett. 71, 1234-1236 (1997).

17. Mahan, G. D., Sofo, J. O. \& Bartkowiak, M. Multilayer thermionic refrigerator and generator. J. Appl. Phys. 83, 4683-4689 (1998).

18. Mahan, G. D. \& Woods, L. M. Multilayer thermionic refrigeration. Phys. Rev. Lett. 80, 4016-4019 (1998).

19. Zeng, T. \& Chen, G. Energy conversion in heterostructures for thermoionic cooling. Microscale Thermophys. Eng. 4, 39-50 (2000).

20. Vashaee, D. \& Shakouri, A. Electronic and thermoelectric transport in semiconductor and metallic superlattices. J. Appl. Phys. 95, 1233-1245 (2004).

21. Shakouri, A., LaBounty, C., Piprek, J., Abraham, P. \& Bowers, J. E. Thermionic emission cooling in single barrier heterostructures. Appl. Phys. Lett. 74, 88-89 (1999).

22. Fan, X. et al. SiGeC/Si superlattice microcoolers. Appl. Phys. Lett. 78, 1580-1582 (2001).
23. LaBounty, C., Shakouri, A. \& Bowers, J. E. Design and characterization of thin film microcoolers. J. Appl. Phys. 89, 4059-4064 (2001).

24. Zhang, J., Anderson, N. G. \& Lau, K. M. AlGaAs superlattice microcoolers. Appl. Phys. Lett. 83, 374-376 (2003).

25. Zhang, J., Anderson, N. G. \& Lau, K. M. Al/sub 0.10/Ga/sub 0.90/As-GaAs microcoolers. IEEE Electron Device Lett. 25, 345-347 (2004).

26. Chao, K. A., Larsson, M. \& Mal'shukov, A. G. Room-temperature semiconductor heterostructure refrigeration. Appl. Phys. Lett. 87, 022103 (2005).

27. Anderson, M. H., Ensher, J. R., Matthews, M. R., Wieman, C. E. \& Cornell, E. A. Observation of bose-einstein condensation in a dilute atomic vapor. Science 269, 198-201 (1995).

28. Jayasekera, T., Mullen, K. \& Morrison, M. A. Evaporative cooling of electrons in semiconductor devices. AIP Conf. Proc. 772, 1279-1280 (2005).

29. Jayasekera, T., Mullen, K. \& Morrison, M. A. Cooling electrons in semiconductor devices: a model of evaporative emission. Phys. Rev. B 75, 035316 (2007).

30. Ray, S. et al. Resonant tunneling transport at $300 \mathrm{~K}$ in GaAs-AlGaAs quantum wells grown by metalorganic chemical vapor deposition. Appl. Phys. Lett. 48, 1666-1668 (1986).

31. Shah, J. Hot electrons and phonons under high intensity photoexcitation of semiconductors. Solid. State Electron. 21, 43-50 (1978).

32. Shah, J., Pinczuk, A., Gossard, A. C. \& Wiegmann, W. Energy-loss rates for hot electrons and holes in GaAs quantum wells. Phys. Rev. Lett. 54, 2045-2048 (1985).

33. White, C. R. H. et al. Optical investigation of a very asymmetric double-barrier resonant-tunneling structure. Phys. Rev. B. 45, 6721-6730 (1992).

34. Suchet, D., Jehl, Z., Okada, Y. \& Guillemoles, J.-F. Influence of hot-carrier extraction from a photovoltaic absorber: an evaporative approach. Phys. Rev. Appl. 8, 034030 (2017).

35. Sadasivam, S., Chan, M. K. Y. \& Darancet, P. Theory of thermal relaxation of electrons in semiconductors. Phys. Rev. Lett. 119, 136602 (2017).

36. Jhalani, V. A., Zhou, J.-J. \& Bernardi, M. Ultrafast hot carrier dynamics in GaN and its impact on the efficiency droop. Nano Lett. 17, 5012-5019 (2017).

37. Zhou, J.-J. \& Bernardi, M. Ab initio electron mobility and polar phonon scattering in GaAs. Phys. Rev. B 94, 201201 (2016).

38. Lake, R. \& Datta, S. Nonequilibrium Green's-function method applied to double-barrier resonant-tunneling diodes. Phys. Rev. B. 45, 6670-6685 (1992).

39. Ashcroft N. W., Mermin N. D. Solid State Physics. (Holt, Rinehart and Winston, 1976).

40. Bescond, M. et al. Thermionic cooling devices based on resonant-tunneling AlGaAs/GaAs heterostructure. J. Phys. Condens. Matter 30, 064005 (2018).

\section{Acknowledgements}

We thank Koung-An Chao for drawing our attention to the concept of thermionic cooling devices. We acknowledge Zacharie Jehl and Daniel Suchet for fruitful discussions, Fabienne Michelini, Laurent Raymond, Nicolas Cavassilas, and Michel Lannoo for numerical support in the quantum transport code implementation, and Ryo Tamaki for assistance on photoluminescence measurements. This work was supported by the Grantin-Aid for Scientific Research from the Japan Society for the Promotion of Science (JSPS) (15H05868, 16F16783, and 19K21957). A.Y. acknowledges the fellowship support from JSPS and MB acknowledges the PEPS Project ICE from "Réseaux Ingénierie verte" of the CNRS.

\section{Author contributions}

A.Y. and T.Y. conducted the experiments. N.N. fabricated the sample. A.Y. analyzed the experimental data, M.B. developed the theoretical modeling, and K.H. supervised the work. All authors were involved in the interpretation and contributed to the final manuscript.

\section{Competing interests}

The authors declare no competing interests.

\section{Additional information}

Supplementary Information is available for this paper at https://doi.org/10.1038/ s41467-019-12488-9.

Correspondence and requests for materials should be addressed to K.H.

Reprints and permission information is available at http://www.nature.com/reprints

Peer Review Information Nature Communications thanks the anonymous reviewers for their contribution to the peer review of this work. Peer reviewer reports are available.

Publisher's note Springer Nature remains neutral with regard to jurisdictional claims in published maps and institutional affiliations. 
(c) Open Access This article is licensed under a Creative Commons Attribution 4.0 International License, which permits use, sharing, adaptation, distribution and reproduction in any medium or format, as long as you give appropriate credit to the original author(s) and the source, provide a link to the Creative Commons license, and indicate if changes were made. The images or other third party material in this article are included in the article's Creative Commons license, unless indicated otherwise in a credit line to the material. If material is not included in the article's Creative Commons license and your intended use is not permitted by statutory regulation or exceeds the permitted use, you will need to obtain permission directly from the copyright holder. To view a copy of this license, visit http://creativecommons.org/ licenses/by/4.0/.

(C) The Author(s) 2019 